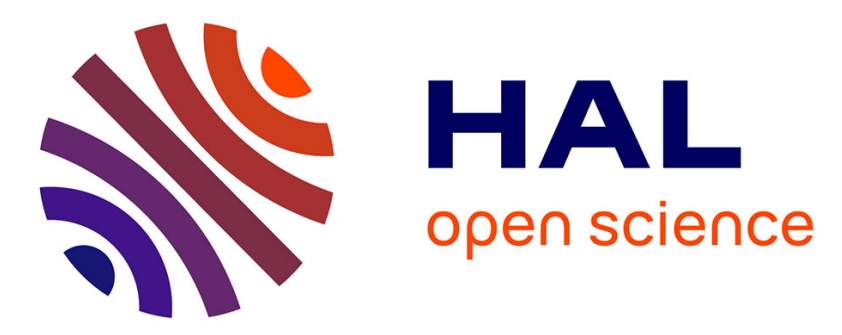

\title{
Kinetics of plaster hydration and structure of gypsum: Experiments and kinetic Monte Carlo simulations with added gypsum seeds
}

\author{
G. Morgado, L. Masurel, Z. Rhodes, R. Lespiat, H. Rétot, A. Lemarchand
}

\section{- To cite this version:}

G. Morgado, L. Masurel, Z. Rhodes, R. Lespiat, H. Rétot, et al.. Kinetics of plaster hydration and structure of gypsum: Experiments and kinetic Monte Carlo simulations with added gypsum seeds. Journal of Crystal Growth, 2019, 507, pp.124-133. 10.1016/j.jcrysgro.2018.11.006 . hal-02612028

\section{HAL Id: hal-02612028 \\ https://hal.science/hal-02612028}

Submitted on 18 May 2020

HAL is a multi-disciplinary open access archive for the deposit and dissemination of scientific research documents, whether they are published or not. The documents may come from teaching and research institutions in France or abroad, or from public or private research centers.
L'archive ouverte pluridisciplinaire HAL, est destinée au dépôt et à la diffusion de documents scientifiques de niveau recherche, publiés ou non, émanant des établissements d'enseignement et de recherche français ou étrangers, des laboratoires publics ou privés. 


\title{
Kinetics of Plaster Hydration and Structure of Gypsum: Experiments and Kinetic Monte Carlo Simulations with Added Gypsum Seeds
}

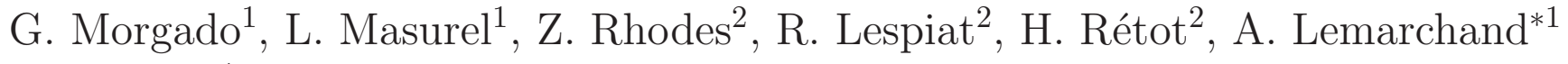 \\ 1 Sorbonne Université, Centre National de la Recherche Scientifique CNRS \\ Laboratoire de Physique Théorique de la Matière Condensée, LPTMC, \\ F-75005 Paris, France \\ 2 Saint-Gobain Research Provence, F-84300 Cavaillon, France
}

*Corresponding author: E-mail: anle@lptmc.jussieu.fr, Tel: +33 (0)1 44274455

Abstract:

Experiments and simulation results are compared in order to make precise the conditions optimizing both the kinetics of plaster hydration and the structure of the resulting material. Calorimetry experiments performed for different amounts of added gypsum seeds are used to assign values to the simulation parameters controlling kinetics, e.g. time step, surface density of gypsum germs on plaster grains. Simulation provides a reliable quantity to follow the beginning of setting, the fraction of gypsum needles belonging to a percolating cluster, as proven by comparing its evolution with the one of the elastic modulus during shear experiments. The simulations reveal that the beginning of needle growth may be hindered by two many small plaster grains, whereas big plaster grains slow down the end of the reaction. We suggest to add gypsum germs with an $m_{g} / m_{p}=0.002$ gypsum-to-plaster mass ratio in order to optimize homogeneity of the final material.

Keywords:

A1. Growth models, A1. Crystal structure, A1. Computer simulation, A2. Industrial crystallization, B1. Calcium compounds 


\section{Introduction}

Optimizing the hydration of plaster represents an economic challenge. To this end, an astonishing variety of additives have been tried in order to control the kinetics of the reaction while improving the mechanical properties of the resulting product $[1,2,3,4,5,6$, $7,8,9,10,11,12,13,14,15,16,17]$. Electrical conductivity $[2,18,19]$, calorimetry [20, 21], acoustic emission [13, 22] and Raman spectroscopy [23] have been used to follow the dynamics of the reaction. The nanoscale structure of the material has been characterized $[24,25]$, including by nuclear magnetic resonance (NMR) [26] and cryogenic transmission electron microscopy [27]. A great deal of effort has been made to establish relationships between the structure and the properties of the material [16, 17, 28, 29, 30, 31, 32, 33]. However, the mechanisms underlying the hydration of plaster are not yet sufficiently well understood for manufacturing conditions to be designed without time-consuming trialand-error tests. The hydration of calcium sulfate hemihydrate leads to the precipitation of calcium sulfate dihydrate according to the reaction:

$$
\mathrm{CaSO}_{4}, \frac{1}{2} \mathrm{H}_{2} \mathrm{O}+\frac{3}{2} \mathrm{H}_{2} \mathrm{O} \rightarrow \mathrm{CaSO}_{4}, 2 \mathrm{H}_{2} \mathrm{O}
$$

In the following, plaster will be used to refer to calcium sulfate hemihydrate and gypsum to calcium sulfate dihydrate. Plaster and gypsum are mainly studied at the macroscopic scale $[18,34,35,36,37]$ or at the atomic scale $[38,39,40,41,42]$.

In order to bridge the gap between these two remote scales, we developed a model which simulates the growth of gypsum needles at the submicrometer scale. We use the tools of statistical physics to account for the interactions of many growing gypsum monocrystals $[19,22,23,43,44]$. When considered at a mesoscopic scale, reaction and diffusion are stochastic, Markovian processes $[45,46]$. The model is based on a small number of stochastic growth processes with well chosen transition rates and has already demonstrated its ability to reproduce the kinetics of plaster hydration, with and without a setting retardant $[23]$.

In order to make the link between a simulation result and a given hypothesis of the 
model without ambiguity, it is necessary to design an as simple as possible model. Although the reaction given in Eq. (1) is exothermic, temperature rise is not sufficient to explain the typical induction time observed in calorimetry experiments. Unavoidable strong assumptions are inherent in the approach and, in particular, temperature variations are ignored. The comparison of simulation results with experiments is crucial to examine the relevance of the hypotheses. The main question is whether the minimal model used so far is sufficient to quantitatively reproduce the addition of an accelerator in the form of micrometer gypsum needles $[16,47]$ for a usual water-to-plaster mass ratio. Our aim is to propose conditions optimizing both the reaction kinetics and the structure of the final material. Special care is taken to compare calorimetry experiments and elastic modulus evolution during plaster hydration with the appropriate quantities provided by the simulation model. Unexpected effects of plaster grain size distribution on reaction speed and crystal structure are interpreted as consequences of steric hindrance during the growth of gypsum needles.

The paper is organized as follows. The simulation model is presented and the choice of the hypotheses are discussed in Section 2. The algorithm is made precise in the Appendix. The comparison of experimental and simulation results is made in Section 3 for kinetics and in Section 4 for properties of the final material. Section 5 is devoted to conclusion.

\section{Model}

Plaster hydration and gypsum precipitation are known to follow a dissolution precipitation mechanism. One of the striking properties of the reaction is the existence of an induction time. Nucleation theory provides an approximate prediction for the rate at which nuclei of a new phase form in the framework of a deterministic approach [49]. Although nucleation theory intends to describe the dynamics of precipitation, it presents arguments based on equilibrium thermodynamics. In particular, a critical crystal size, related to the maximum of Gibbs free energy, is introduced and interpreted as a limit size between 
redissolution and further growth of the crystal according to Ostwald ripening. Hence, following nucleation theory would imply the time-consuming simulations of germs which first grow and then disappear. In addition, nucleation theory ignores the description of diffusion in the liquid phase.

We have built a minimal model relying on purely kinetic arguments and that has already been checked by comparison with conductimetry [19], acoustic emission [22] and dynamic Raman spectroscopy [23] experiments. The simplest way to account for an induction period by elementary processes is to imagine an autocatalytic process: As reaction proceeds, the product acts as its own catalyst, exactly as in polymerisation, in which the production of intermediates interacting with the monomers accelerates their further aggregation to the chain. This observation led us to assume the presence of existing small germs, first growing at a rate proportional to their surface. Indeed, at a time scale smaller than the simulation time step, needle growth begins with local precipitation of gypsum on the needle surface. When a random deposit of gypsum appears on the needle, it creates a defect on its surface. This defect spreads out over the surface, allowing the needle to recover its equilibrium shape. The spreading time is proportional to the surface $s$ of the needle. A larger needle will therefore keep an out-of-equilibrium shape longer than a smaller one. For a given period of time, an out-of-equilibrium needle surface will see more defects appear than a needle surface at equilibrium. Following these arguments, we arrived to the reasonable assumption according to which a needle with a larger surface aggregates a larger amount of gypsum and grow faster. Moreover, the temperature rise during the reaction contributes to its acceleration and autocatalytic nature. Admitting that a bigger needle grows faster reproduces this phenomenon in an effective way. In the simulations, the needles never shrink but can remain a long time before being randomly chosen as candidates for growing. They may be already blocked by the surrounding needles and never grow, which accounts for Ostwald ripening in an effective way. 
An inital growth probability proportional to the surface induces the autocatalytic growth of the subcritical needles, which ensures the existence of the induction time observed in plaster hydration: Small germs slowly grow and, as their surface area increases, they grow faster and faster. Such a rule would lead to the explosion of growth rates. However, as the probability of growth increases with the surface, the growth of the needle becomes controlled by the arrival time of the calcium and sulfate ions in its vicinity. Diffusion of the dissolved ions toward a given gypsum face is hindered in the tortuous pores left between entangled needles. Reaction becomes faster whereas diffusion is slowed down all the more when a certain number of needles are already well formed. The two regimes cross when the needle reaches a critical surface $s_{c}$, which presents analogies with the critical size introduced in nucleation theory. The sensitivity of the simulation results to the critical surface has been discussed in reference [22] and the value of $s_{c}$ has been chosen by comparing optical microscopy images during the reaction and simulation results.

According to the Wulff theorem, equilibrium crystal growth is ruled by minimization of the surface energy [48]. Assuming local equilibrium would imply that, at each time step, a needle reaches the shape that minimizes its surface energy. Respecting the ratio of the surface tensions between the basal and lateral faces of a gypsum crystal means that the small square face of length $w$ grows $\alpha$ times faster than the long rectangular face of length $l$, in order to maintain the aspect ratio $\alpha=l / w$ of a needle constant. Scanning electron microscopy images of the material revealed that the aspect ratio $\alpha$ is close to 12 in average but with an important spreading of the sizes of the different gypsum needles [23]. In order to take this phenomenon into account, we suppose that a needle keeps a parallelepipedic shape but with a variable aspect ratio $\alpha$ in the interval $[5,19]$. In addition, we found that imposing too small an interval of $\alpha$ values may lead to growth blocking and incomplete reaction .[23]

An important hypothesis on which our model relies is that the speed of the reaction is 
imposed by gypsum precipitation. We refer to the conductivity experiments and the analysis of Amathieu and Boistelle [18] which show that, if one excepts the very beginning and the end of the reaction, the solution remains saturated with respect to hemihydrate and supersaturated with respect to dihydrate. Indeed, as plaster is introduced in water, conductivity nearly instantaneously reaches a constant value, proving that plaster dissolves extremely fast compared to gypsum crystal growth. As gypsum precipitates, plaster dissolves almost instantly so that the concentration remains at the saturation value, as long as there is enough plaster left. The approximation is analogous to the standard adiabatic hypothesis or the quasisteady-state approximation, which assumes that the fast variable, here the concentration of plaster in water, reaches a quasisteady state. The fast variable adapts to the variations of the slow variable, here the amount of precipitated gypsum. Gypsum precipitation drives the reaction and plaster dissolution adiabatically follows gypsum precipitation.

The simulation is based on a kinetic Monte Carlo algorithm simulating random processes of gypsum needle growth and plaster grain dissolution $[19,22,23,43,44]$. An initial configuration of spherical plaster grains with nucleation sites and possibly gypsum seeds in suspension is generated. The algorithm consists of a sequence of two steps: First, the growth of each needle is considered with the appropriate probability and performed if steric hindrance and aspect ratio conservation permit it. Second, the shrinking of each grain is completed, according to the conservation of matter. The number of needles and their orientation are fixed. Needles and grains are immobile. After a succession of growth and dissolution steps, the simulation ends either when the total amount of plaster has dissolved or when all the needles are blocked by steric hindrance. A more detailed description of the initial condition and the algorithm is given in the Appendix. Table I lists the parameters that characterize plaster grains and their values in the simulations. Table II provides the parameter values for both types of gypsum needles. 


\begin{tabular}{|c|c|}
\hline \multicolumn{2}{|c|}{ Plaster grains } \\
\hline density & $\rho_{p}=2.63 \times 10^{3} \mathrm{~kg} \cdot \mathrm{m}^{-3}$ \\
molar mass & $M_{p}=0.145 \mathrm{~kg}$ \\
\hline \multicolumn{2}{|c|}{ Initially } \\
\hline $\begin{array}{c}\text { water-to-plaster mass ratio } \\
\text { number of grains } \\
\text { grain radius }\end{array} m_{w} / m_{p}=0.8$ \\
\multicolumn{2}{|c|}{ At time $t$} \\
\hline \multicolumn{2}{|c|}{$0.222 \mu \mathrm{m} \leq R_{0} \leq 30 \mu \mathrm{m}$} \\
\hline number of grains \\
grain radius \\
radius decrease during $\Delta t$
\end{tabular}

Table 1: Parameters characterizing the plaster grains and their values in the simulations. The size of the simulation box is fixed at $L=114 \mu \mathrm{m}$.

\begin{tabular}{|c|c|c|}
\hline & Gypsum needles on plaster grains & Added gypsum needles in water \\
\hline $\begin{array}{c}\text { density } \\
\text { molar mass }\end{array}$ & \multicolumn{2}{|c|}{$\begin{array}{c}\rho_{g}=2.32 \times 10^{3} \mathrm{~kg} \cdot \mathrm{m}^{-3} \\
M_{g}=0.172 \mathrm{~kg}\end{array}$} \\
\hline \multicolumn{3}{|c|}{ Initially } \\
\hline $\begin{array}{c}\text { length } \\
\text { aspect ratio } \\
\text { critical surface } \\
\text { number }\end{array}$ & $\begin{array}{c}\text { surface density: } \\
g=0.08 \mu \mathrm{m}^{-2} \text { for } R_{0}>1 \mu \mathrm{m} \\
\text { fraction of small grains bearing one germ: } \\
\phi=0.5 \text { for } R_{0} \leq 1 \mu \mathrm{m} \\
l_{0}=0.024 \mu \mathrm{m} \\
\alpha_{0}=12 \\
s_{c}=0.2 \mu \mathrm{m}^{2} \\
n\end{array}$ & $\begin{array}{c}\text { gypsum-to-plaster mass ratio: } \\
\begin{array}{c}0 \leq m_{g} / m_{p} \leq 0.002 \\
\\
l_{0}^{\prime}=1 \mu \mathrm{m} \\
\alpha_{0}^{\prime}=12 \\
s_{c}^{\prime}=0.2 \mu \mathrm{m}^{2} \\
n^{\prime}\end{array}\end{array}$ \\
\hline \multicolumn{3}{|c|}{ At time $t$} \\
\hline $\begin{array}{c}\text { length } \\
\text { width } \\
\text { aspect ratio } \\
\text { surface } \\
\text { growth probability } \\
\text { volume increase per free } \\
\text { surface during } \Delta t\end{array}$ & $\begin{array}{c}l \\
w \\
\alpha=l / w=12 \pm 7 \\
s \\
p s / s_{c} \text { if } s<s_{c} \\
p=0.1 \text { if } s \geq s_{c} \\
w^{2} \Delta l_{0} \text { with } \\
\Delta l_{0}=0.03 \mu \mathrm{m}\end{array}$ & $\begin{array}{c}l^{\prime} \\
w^{\prime} \\
\alpha^{\prime}=l^{\prime} / w^{\prime}=12 \pm 7 \\
s^{\prime} \\
p s^{\prime} / s_{c} \text { if } s^{\prime}<s_{c} \\
p=0.1 \text { if } s^{\prime} \geq s_{c} \\
w^{\prime 2} \Delta l_{0}^{\prime} \text { with } \\
\Delta l_{0}^{\prime}=0.03 \mu \mathrm{m}\end{array}$ \\
\hline
\end{tabular}

Table 2: Parameters characterizing the gypsum needles on plaster grains and added in suspension and their values in the simulations. 
The simulation algorithm is able to generate an initial configuration of plaster grains within a cubic box according to the grain size distribution used in the experiments.

The grain size distribution used in the experiments is specific: It is very large and corresponds to a powder with a big amount of small grains and a few big grains. Our goal is twofold. We first wish to check if the model is able to reproduce experiments leading to the challenging generation of such a very large number of grains and then to analyze the consequences of the specificities of the distribution on kinetics and microstructure. Specifically, the considered grain size distribution contains both a large number of small grains, with radii in the order of $0.25 \mu \mathrm{m}$, and a small number of large grains with radii up to $30 \mu \mathrm{m}$. This type of grain size distribution poses specific problems because it requires the simulation of a very large number of objects, grains and needles. For a single large grain of radius $R=30 \mu \mathrm{m}$, the number of small grains almost reaches $10^{5}$. In order to use a reasonable computing memory size, we have chosen to generate configurations containing $N_{0}=10^{5}$ grains and only one grain of radius $R=30 \mu \mathrm{m}$. We have selected a usual value of the water-to-plaster mass ratio, $m_{w} / m_{p}=0.8$. Imposing the number of grains and the water-to-plaster mass ratio sets the size of the simulation box. For the chosen values of $N_{0}$ and $m_{w} / m_{p}$, we obtain a cubic simulation box of side length $L=114 \mu \mathrm{m}$. Generating the plaster grain configuration does not require any packing algorithm, provided that the grains are placed in decreasing radius order. A typical example of an initial configuration of plaster grains generated by the algorithm is shown in Figure 1a.

Typical values of needle length and aspect ratio have been derived from the analysis of images of gypsum crystals obtained by electron microscopy. Dynamic Raman spectroscopy has been used to follow the kinetics of the reaction for different water-to-plaster ratios, $m_{w} / m_{p}$, with and without addition of citric acid [23]. The amount of germs on the plaster grains is not easily determined experimentally. We introduce two parameters in order to control this quantity, the surface density $g$ of needles on large grains (with a radius larger than $1 \mu \mathrm{m}$ ) and the fraction $\phi$ of small grains (of radius $R_{0}<1 \mu \mathrm{m}$ ) bearing 


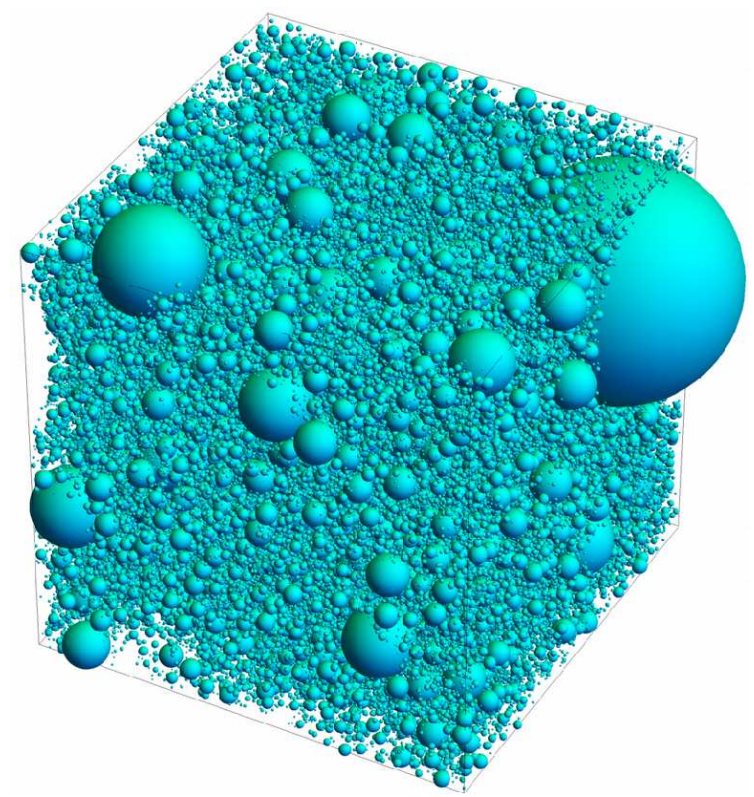

(a)

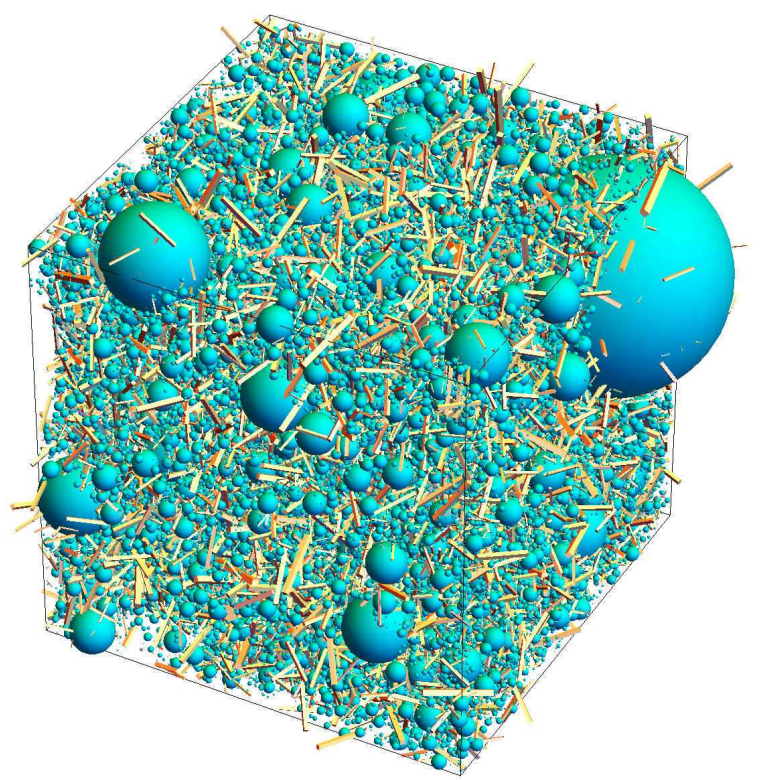

(b)

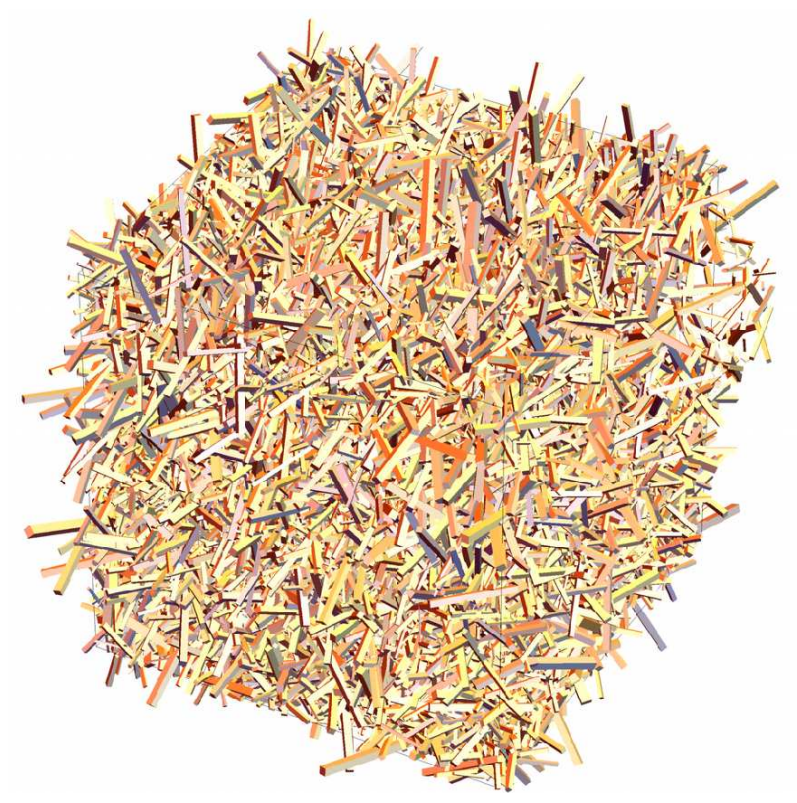

(c)

Figure 1: (a) Initial, (b) intermediate, and (c) final configurations obtained in a simulation of $10^{5}$ plaster grains, without gypsum seeds in suspension, for the parameter values listed in Tables I, II. Plaster grains are represented by blue spheres and gypsum needles by orange parallelepipeds. The intermediate configuration is obtained when the extent of the reaction reaches $10 \%$. 
one germ. The choice of these parameter values will be discussed in the next section. For a surface density of needles $g=0.08 \mu \mathrm{m}^{-2}$ on large grains and for a fraction $\phi=0.5$ of small grains with a single germ, the number of needles on grains is about 70,000 per simulation box. With the addition of gypsum germs in suspension, the total number of needles can reach 220,000 for a gypsum-to-plaster mass ratio $m_{g} / m_{p}=0.002$. Figure $1 \mathrm{~b}$ shows the intermediate configuration obtained when the extent of the reaction reaches $10 \%$ and Figure 1c shows the final configuration after complete dissolution of the plaster grains and growth of the gypsum needles. The latter form a very entangled skeleton. It can be observed that the disappearance of the largest plaster grain results in the formation of a pore in the final configuration.

We used the simulation algorithm to study the kinetics of plaster hydration and the structure of the final material. Next section is devoted to the presentation of the simulation results obtained in kinetics and their comparison with experimental results. Some parameters of the simulation are not independent and the choice of their value is arbitrary. In particular, the choice of the constant growth probability $p$ and the length increase $\Delta l_{0}$ during the time step $\Delta t$ fixes the time step, which is determined by comparison with kinetics experiments. Doubling the product $p \Delta l_{0}$ would simply lead to doubling the time step $\Delta t$.

\section{Kinetics}

\subsection{Extent of the reaction}

From an experimental point of view, the easiest way to follow the extent of the reaction is to exploit its exothermicity and measure the temperature rise of the sample. Figure $2 \mathrm{a}$ shows the results obtained for the grain size distribution used in Figure 1 and for different amounts of added germs of micrometer length. The black dotted line obtained without germs in suspension shows a drop in temperature between $600 \mathrm{~s}$ and $2600 \mathrm{~s}$, indicating poor sample insulation during the reaction and thus inducing a delay in precipitation 
and a shift of the curve to the right. However, the results are in line with expectations: Adding gypsum germs tends to speed up the reaction.

The simulation gives access to the extent $\xi$ of the reaction by calculating the total undissolved plaster volume at time t:

$$
\xi=1-\frac{V_{p}(t)}{V_{p}(t=0)}
$$

If the reaction is not complete, $\xi$ does not tend towards 1 , as observed at lower gypsumto-plaster mass ratios. Simulation results for the extent of the reaction are given in Figure 2b. The predicted results are qualitatively consistent with the calorimetry experiments shown in Figure 2a. The simulation results obtained without gypsum germs in suspension (black curve) satisfactorily reproduce the induction time of the reaction and its further runaway. This typical appearance of an autocatalytic reaction is obtained by the choice of needle growth probability, proportional to their surface. In the simulation, the reaction speed is limited by crystallization. Addition of germs increases gypsum surface area on which precipitation can occur and therefore leads to an acceleration of the reaction. This result confirms the hypothesis that the dissolution of plaster is not limiting. According to experiments, the initial length of the added germs is fixed at $l_{0}^{\prime}=1 \mu \mathrm{m}$. The clear acceleration of the reaction observed in Figure 2 for an as small gypsum-to-plaster mass ratio as $m_{g} / m_{p}=0.0001$ indicates that the surface of the added germs is larger than the critical surface value $s_{c}$. For square-based parallelepipeds, the initial surface can be linked to the length as follows:

$$
s^{\prime}(t=0)=4 l_{0}^{\prime} w_{0}^{\prime}+2 w_{0}^{\prime 2}=2 \frac{l^{\prime 2}}{\alpha_{0}^{\prime}}\left(2+\frac{1}{\alpha_{0}^{\prime}}\right)=0.35 \mu \mathrm{m}^{2}
$$

Consistently, the critical surface obeys $s_{c}=0.2 \mu \mathrm{m}^{2}$.

It is customary to consider three characteristic times denoted by $t_{10}, t_{50}$, and $t_{90}$ after which $10 \%, 50 \%$ and $90 \%$ of the reaction took place, respectively. The induction time $t_{10}$ is often considered to give an order of magnitude of the transition time between a liquid 


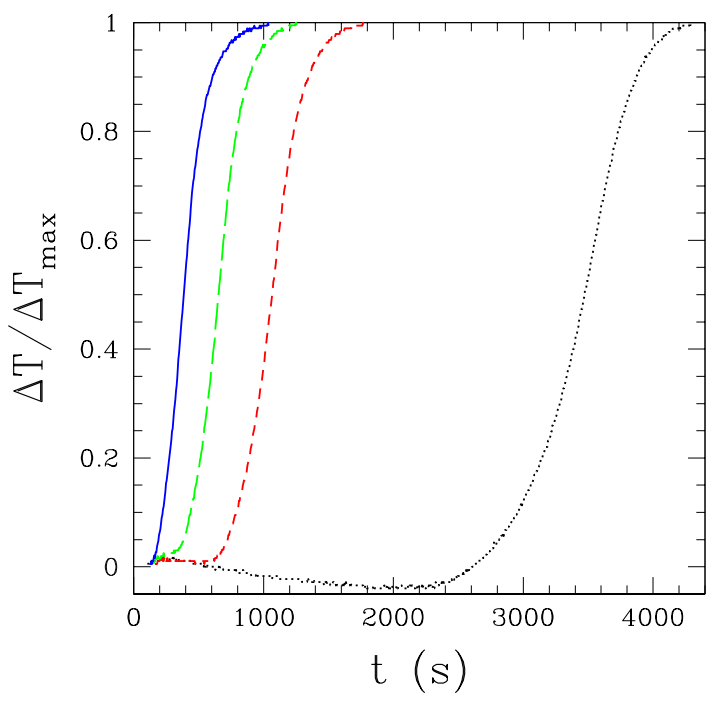

(a)

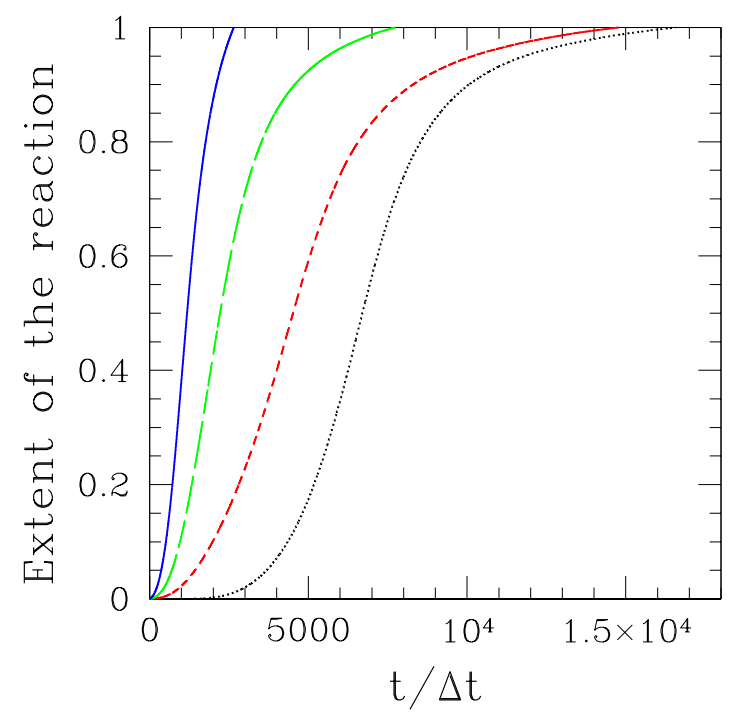

(b)

Figure 2: (a) Time evolution of normalized temperature rise in calorimetry experiments for the water-to-plaster mass ratio $m_{w} / m_{p}=0.8$, without gypsum germs in suspension $m_{g} / m_{p}=0$ (black dotted line), with gypsum germs for different gypsum-to-plaster mass ratios $m_{g} / m_{p}=0.0001$ (short-dashed red line), $m_{g} / m_{p}=0.0005$ (long-dashed green line), $m_{g} / m_{p}=0.002$ (solid blue line) and (b) extent of the reaction deduced from the simulations for the grain size distribution illustrated in Figure 1, the same values of $m_{w} / m_{p}$ and $m_{g} / m_{p}$ as in the experiments, and the parameter values given in Tables I, II. 
and a paste. However, hardening of the material is evidenced by the sudden increase in the elastic modulus during a shear experiment and is not straightforwardly related to the value of the extent of the reaction. The time $t_{50}$ evaluates the time at which the material acquired sufficient mechanical properties to be cut. The reaction can be considered complete at the time $t_{90}$.

The experimental and simulation results for these three characteristic times are compared in Figure 3 . The induction times $t_{10}$ are correctly predicted by the simulations when germs are added. By adjusting the curves, a physical value can be assigned to the simulation time step. The value $\Delta t=2.2 \mathrm{~s}$ is found by adjusting experimental and simulation results for $t_{10}$ and $m_{g} / m_{p} \geq 0.0005$. However, the prediction of the simulation for $t_{10}$ without added germs is smaller than the experimental results, partly because of the artificial slowing down of the experiment due to poor sample insulation but also possibly due to some hypotheses of the model. Before calling the model into question, we first try to optimize the choice of parameter values. In order to reproduce a clear difference between the induction times without and with added germs, the initial length $l_{0}$ of the germs at the surface of the grains has to be chosen as small as possible. We fixed $l_{0}=0.024 \mu \mathrm{m}$, which corresponds to needles of width $w_{0}=2 \mathrm{~nm}$. This result differs from experimental values [42]. The model, valid at the submicrometer scale is not supposed to reveal material properties at the nanoscale. However, the values of $l_{0}$ and $w_{0}$ chosen in the simulations account for macroscopic kinetics in an effective way.

The discrepancy between the calorimetry results and the simulation results without added germs may also suggest that the number of germs on grains was overestimated in the simulation. In fact, the parameter $g$ which fixes the surface area of embryonic needles on big grains (of radius $R_{0}>1 \mu \mathrm{m}$ ) and the fraction $\phi$ of small grains (of radius $R_{0}<1 \mu \mathrm{m}$ ) bearing one germ were empirically chosen, without any direct experimental validation being possible. The strength of the simulation is to propose an estimation of these pa- 
rameters. We checked different values of $g$ and $\phi$ in the range $0.01 \mu \mathrm{m}^{-2} \geq g \geq 0.1 \mu \mathrm{m}^{-2}$ and $0.5 \geq \phi \geq 1$. Switching from $\phi=1$ (one germ on each plaster grain of radius $R_{0}<1 \mu \mathrm{m}$ ) to $\phi=0.5$ (50\% of grain of radius $R_{0}<1 \mu \mathrm{m}$ bear one germ) does not change the kinetics significantly: The germs on the grain are initially much smaller than the added germs in suspension. Removing $50 \%$ of germs on the small grains does not slow down the reaction much. However, a further decrease in $\phi$ leads to an incomplete reaction, especially if the water-to-plaster mass ratios $m_{w} / m_{p}$ is decreased, in disagreement with experimental facts. Decreasing $g$ and using the time step value deduced from the tuning of simulation and experimental induction times $t_{10}$ in the case of added germs does not lead to a significant improvement of the agreement between the simulation and experimental values of $t_{10}$ without added germs. This point can be justified by the small number of big grains with respect to the very large number of small grains in the considered grain size distribution. On the other hand, decreasing $g$ significantly slows down the end of the reaction without added germs: Actually, the needles first develop in the place initially occupied by water and space is then freed by the gradual dissolution of the grains. The big grains are the last to be dissolved and the growth speed at the end of the reaction is controlled by the centripetal growth of the needles on the big grains. A too small value of $g$ leads to a too small final reaction speed and even to an incomplete reaction. This trend is already visible in Figure 3 for $g=0.08 \mu \mathrm{m}^{-2}$, whereas the calorimetry curves obtained with and without added germs seems to be translated without deformation. In summary, the comparison between the simulations and the experiments allows us to propose $g=0.08 \mu \mathrm{m}^{-2}$ for the surface density of needles on big grains and a fraction $\phi=0.5$ of small grains bearing one germ as reasonable values. The parameters $g$ and $\phi$ fix the number of needles that actually grow. The larger $g$ and $\phi$, the more needles, therefore smaller due to the conservation of matter. The model can be used to determine $g$ and $\phi$ in other experimental conditions, including the use of additives and treatments of the plaster grains which modify the surface energies of gypsum crystal faces [19]. Small values of $g$ and $\phi$ correspond to gypsum material composed of a small number of big needles, with 


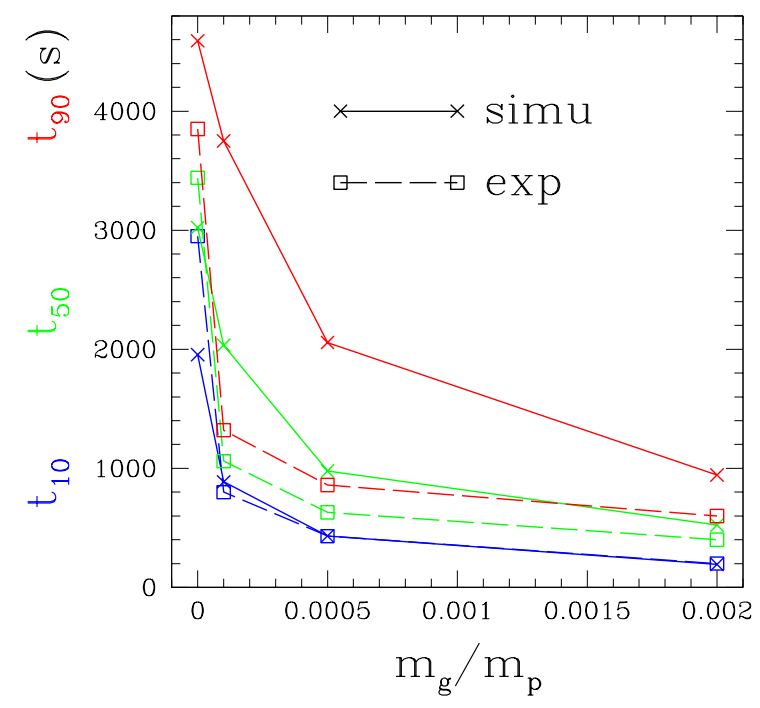

Figure 3: Time $t_{x}$ after which $x=10 \%$ (blue), $50 \%$ (green), and $90 \%$ (red) of the reaction occurred when gypsum germs are added in suspension as a function of gypsum-to-plaster mass ratio $m_{g} / m_{p}$. Squares and dashes: Experimental results deduced from Figure 2a. Crosses and solid lines: Simulation results deduced from Figure $2 \mathrm{~b}$ by choosing $\Delta t=2.2$ s.

big pores potentially reducing the resistance to denting. On the contrary, a large number of smaller, entangled needles increases the number of contacts between the needles and a priori improves mechanical properties. Hence, the simulation results offer a criterion relating the features of the initial plaster powder and the final structure of the gypsum material.

The results prove that a minimum model, introducing an effective mean density of preexisting germs and autocatalytic growth rules, is able to reproduce some macroscopic trends of gypsum precipitation kinetics. Nevertheless, the agreement is not perfect. The simulation model could certainly be improved by removing the hypothesis on the preexistence of germs on the surface of the grains and by introducing growth rules according to nucleation theory, which could result in a larger delay for the beginning of the reaction without added germs $[49,50,51]$. 


\subsection{Evolution of mechanical properties during reaction}

In order to measure the elastic shear modulus at the beginning of the reaction, blades with a rotation motion of very low amplitude and frequency are plunged in the mixture and extracted before the end of the setting. The results are given in Figure 4a. There is a sudden increase in the elastic shear modulus, which occurs sooner if germs are added. The sudden increase corresponds to the start of stiffening of the material.

When comparing the shear experiments and the calorimetry experiments, we state that, for example for $m_{g} / m_{p}=0.0001$, the time at which the elastic modulus abruptly increases differs by about $30 \%$ from the time $t_{10}$ at which the extent of the reaction reaches 0.1: A faster setting is not straightforwardly associated with a faster precipitation of gypsum. It is more appropriate to compare the stiffening time deduced from the shear experiments with the percolation time of the needles in the simulations. The evolution of the fraction of needles belonging to a percolating cluster, i.e., forming a continuous path between two opposite sides of the simulation box, is shown in Figure 4b. The percolation time is defined as the time when a first percolating cluster appears. The intermediate configuration given in Figure 1, when $10 \%$ of the reaction has taken place, gives an image of the beginning of percolation. Visually, we state that the onset of setting occurs at the very beginning of the reaction. As shown in Figure 5, the agreement between the experimental stiffening time and the percolation time predicted by the simulations is excellent if one excepts the small delay in the experiments without added germs. Very satisfactorily, the values of the time step found by comparing experiments and simulations for chemical properties in Figure 2 and mechanical properties in Figure 5 are the same. Qualitatively, we retain that setting is related to a mechanical property and corresponds to the moment where gypsum needles form a rigid skeleton, which may arise for different values of the extent of the reaction. The latter is associated with reaction kinetics. The two following extreme examples illustrate that, depending on plaster grain size distribution, needle percolation may correspond to different amounts of precipitated gypsum. Without added 


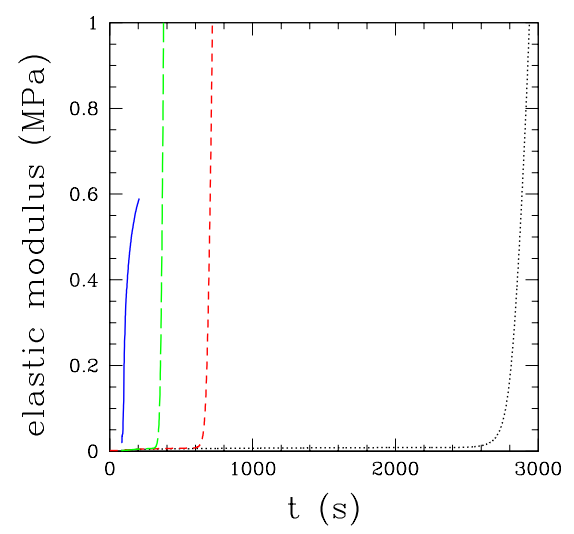

(a)

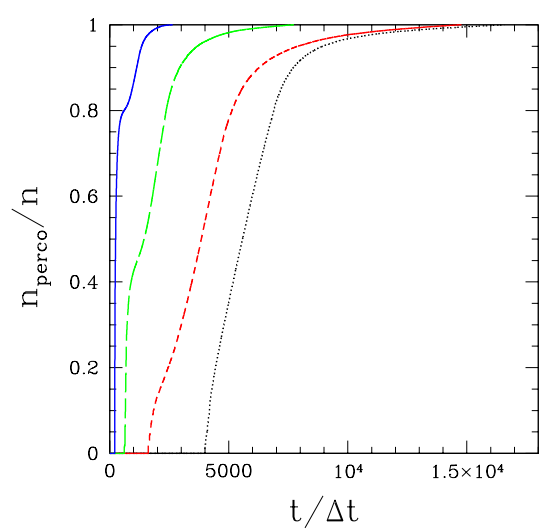

(b)

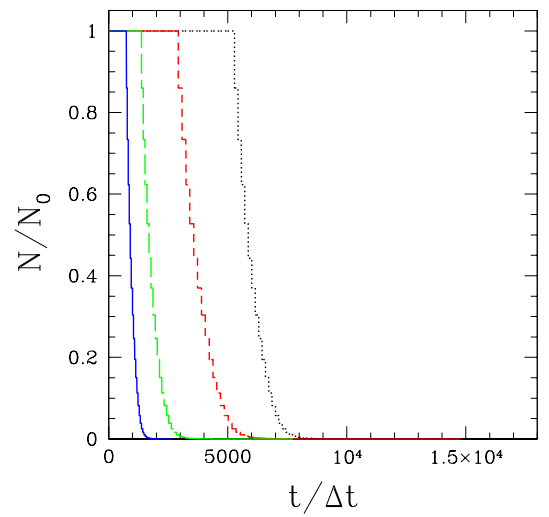

(c)

Figure 4: Time evolutions of (a) the elastic modulus in shear experiments, (b) the normalized number $n_{\text {perco }} / n$ of needles belonging to a percolating cluster deduced from the simulations, and (c) the normalized number $N / N_{0}$ of undissolved plaster grains in the simulations for different gypsum-to-plaster mass ratios (see caption of Figure 2.). 


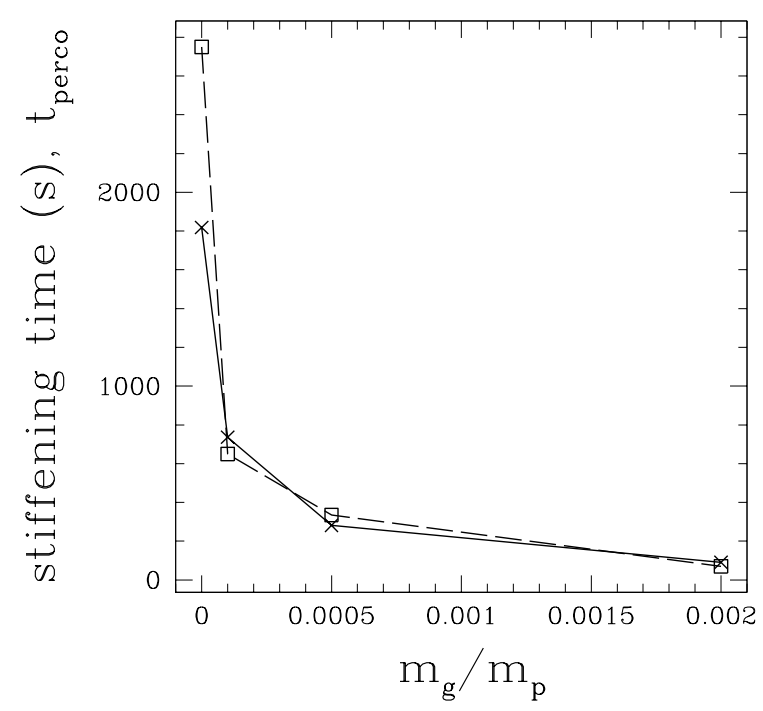

Figure 5: Characterization of setting dynamics for different gypsum-to-plaster mass ratios $m_{g} / m_{p}$. Squares and dashed line: Stiffening time deduced from the experimental results given in Figure 4a. Crosses and solid line : Percolation time deduced from the simulation results given in Figure $4 \mathrm{~b}$ by choosing $\Delta t=2.2 \mathrm{~s}$.

needles and for a small number of big plaster grains and no small plaster grains in the initial distribution, the mean distance between grains is large. Consequently, the mean distance between germs located on the surface of the grains is also large. Hence, needles need to sensitively grow to cover at least half the mean distance between two grains to get in contact. A large amount of gypsum has to precipitate before percolation threshold is reached. Setting occurs for a larger extent of the reaction. On the contrary, with added needles or many small plaster grains bearing germs, percolation will be reached for a smaller amount of precipitated gypsum. Percolation depends on a structural property, the mean distance between initial germs, and not only on chemical kinetics.

The number of needles belonging to the first percolating cluster strongly depends on the number of added germs. The more germs are added, the larger the first percolating cluster is. According to Figure 4c, it contains up to $65 \%$ of the needles for $m_{g} / m_{p}=0.002$, about $35 \%$ of the needles are rapidly aggregated for $m_{g} / m_{p}=0.0005$, and less than $5 \%$ of the needles form the first percolating cluster for $m_{g} / m_{p}=0.0001$. After the sudden 
formation of the first percolating cluster, needles are more slowly aggregated. The speed of cluster formation in Figure $4 \mathrm{~b}$ is compared with the change in the number of plaster grains in Figure 4c. Due to discretization of grain radii, the curves given in Figure 4c show steps that reveal the sudden disappearance of a class of grains of the same radius. The first decrease in the number of plaster grains in Figure 4c can be correlated with the second accelerating phase of percolating cluster growth in Figure 4b: The smallest plaster grains dissolve and disappear first, freeing up space for the needles to grow and aggregate. The early growth of the percolating cluster is thus slowed down by the presence of small plaster grains. It is worth noting that the model predicts that for the chosen parameter values, all the needles belong to a percolating cluster at the end of the reaction. Choosing $m_{w} / m_{p}=0.8$ for the water-to-plaster mass ratio clearly ensures sufficient cohesion between all gypsum crystals.

Hence, the number of needles belonging to a percolating cluster is a good quantity to characterize the evolution of the mechanical properties during plaster setting. Contrary to the shear experiments that have to be stopped just after the beginning of setting, the growth of the percolating cluster in the simulations can be followed until the end of the reaction and offers unexpected predictions. The simulations predict steric hindrance and a slower start of material hardening due to the presence of a large number of small plaster grains. At the end of the reaction, the slower growth of percolating clusters can be attributed to the steric hindrance generated by the big grains, which are the last to dissolve. The effect is all the more pronounced as the ratio between the radii of large and small grains is important.

\section{Structure of the final material}

After studying the influence of the addition of germs on reaction dynamics, we are now interested in its influence on the morphology of the resulting material. Figure 6 shows the normalized distributions of needle lengths $l$ obtained by simulation for different amounts 


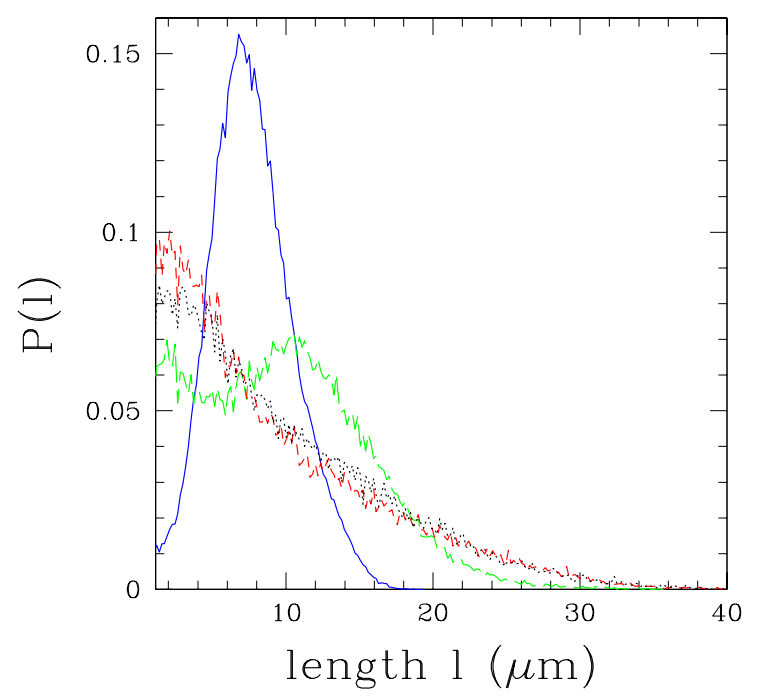

Figure 6: Distribution $P(l)$ of the lengths $l$ of gypsum needles in the simulated material, for different values of gypsum-to-plaster mass ratios, $m_{g} / m_{p}=0$ (black dotted line), $m_{g} / m_{p}=0.0001$ (red short-dashed line), $m_{g} / m_{p}=0.0005$ (green long-dashed line) and $m_{g} / m_{p}=0.002$ (blue solid line).

$m_{g} / m_{p}$ of added germs. The normalized distributions are calculated for needles of length $l>1.1 \mu \mathrm{m}$, i.e., having sufficiently grown. Indeed, the added germs have an initial size $l^{\prime 0}=1 \mu \mathrm{m}$ and those present on plaster grains initially measure $l_{0}=0.024 \mu \mathrm{m}$.

Without added germs, the proportion of needles not exceeding $l=1.1 \mu \mathrm{m}$ is $40 \%$. This result confirms that the selected value of the surface density $g$ may be overestimated. However, a smaller value would have led to the same final structure, as the needles that remained embryonic are less likely to inhibit the growth of others. Surprisingly, the choice of too high a value for $g$ still leads to the simulation of a correct structure. As a reminder, needles grow at a rate proportional to their surface area below the critical surface area $s_{c}=0.2 \mu \mathrm{m}^{2}$ and at a constant rate above $s_{c}$. By analogy with Eq. (3) the critical surface can be linked to the critical length $l_{c}$ as follows:

$$
s_{c}=2 \frac{l_{c}^{2}}{\langle\alpha\rangle}\left(2+\frac{1}{\langle\alpha\rangle}\right)
$$


which leads to:

$$
l_{c}=\sqrt{\frac{s_{c}\langle\alpha\rangle^{2}}{2(1+2\langle\alpha\rangle)}}
$$

For the values given in Table II, we find $l c=0.76 \mu \mathrm{m}$. The germs on grains are therefore subcritical, while the added germs are over-critical. Without added germs $\left(m_{g} / m_{p}=0\right)$, the first germs on grains that grow and reach critical length are likely to become very large as they are not disturbed by the other still embryonic needles. This leads to a very wide $P(l)$ distribution, with many micrometer-sized needles and some needles with large lengths (up to $40 \mu \mathrm{m}$ ). As a result, the material is heterogeneous, suggesting mechanical weaknesses.

The situation changes little for $m_{g} / m_{p}=0.0001$ and 0.0005 but is radically different for $m_{g} / m_{p}=0.002$. In the latter case, the distribution is very sharp around $l_{\max }=7.5 \mu \mathrm{m}$. This value can be estimated using the simulation results and conservation of matter. In a simulation run, a total number $n_{t o t}=158897$ of needles of length greater than $1.1 \mu \mathrm{m}$ are generated in a volume $V_{g}=473556 \mu \mathrm{m}^{3}$. Considering that the needle length distribution is peaked, we assume that all the needles have a length equal to $l_{\max }$ and find:

$$
l_{\max }=\left(\frac{V_{g}\langle\alpha\rangle^{2}}{n_{t o t}}\right)^{1 / 3}=7.5 \mu \mathrm{m}
$$

for $\langle\alpha\rangle^{2}=12$, which perfectly agrees with the maximum of the peak for $m_{g} / m_{p}=0.002$ in Figure 6. In this case, the proportion of added needles reaches 70\%. As these needles are overcritical, they always grow at a constant rate, which favors the homogeneity of their final sizes. As shown in Figure 6, there are no large needles with a length greater than $19 \mu \mathrm{m}$ and the proportion of needles that have not grown is small. This raises the question of whether, by adding more germs, the distribution would not be even more peaked. Obtaining a more homogeneous material reduces the risk of defect generation and a priori improves the mechanical properties. Howewer larger $m_{g} / m_{p}$ values simultaneously lead to shorter induction times, preventing from spreading plaster paste on the support. The $m_{g} / m_{p}$ value can therefore not exceed a certain level. Among the values evaluated in this study, $m_{g} / m_{p}=0.002$ provides the best properties. 


\section{Conclusion}

We used the model of plaster hydration that we developed to analyze the effect of adding gypsum germs in suspension on kinetics and structure of the material. We have checked the ability of our kinetic Monte Carlo algorithm to reproduce experimental results showing the acceleration of gypsum crystallization when micrometer gypsum needles are initially added to water. The agreement between the evolution of the extent of the reaction deduced from the simulations and the temperature rise deduced from calorimetry experiments, on one hand, and the evolution of the ratio of needles belonging to percolating clusters in the simulations and the experimental measurements of the elastic modulus of the material, on the other hand, validates the model. The main hypotheses on needle growth dynamics have been confirmed and physical values have been assigned to the parameters of the model. By comparing kinetics with and without added germs, the model allows an evaluation of the surface density of gypsum germs on plaster which is not easily available from experiments. The model relies on the hypothesis according to which the driving process of the reaction is gypsum precipitation adiabatically followed by plaster dissolution. Initial needle growth probability is assumed to be proportional to needle surface. At the beginning of the reaction and in the absence of steric hindrance, simulations show that the speed of the reaction is controlled by the total surface on which gypsum can precipitate, i.e., on the number and size of existing nucleation sites and their aspect ratio.

The simulation results obtained when gypsum seeds are initially added highlight that choosing an additive on the basis of its effects on reaction speed when the goal is to obtain a faster setting can be misleading [16]. After the limit of the elastic domain has been exceeded, the material can still evolve and reaction may be far from being finished. The extent of the reaction should not be confused with the evolution of the mechanical properties of the material. Instead, we have shown that the abrupt increase of the shear modulus in the experiments perfectly correlates with the formation of the first percolating 
cluster of needles in the simulations. The increase of the amount of added gypsum germs has been related to the modification of the final structure. For a sufficient amount of added germs, the distribution of needle length becomes narrow. Needles with analogous sizes imply a more homogeneous material. This feature reduces the risk of creating local defects that could be at the origin of cracks [52].

Besides, the simulations show that a wide range of grain radii and a very large number of small grains may induce a slowing down of the reaction. Many small plaster grains are a source of steric hindrance which slows needle growth at the beginning of the reaction, but rapidly disappears as the grains are dissolved. Another source of steric hindrance is due to very big grains taking a very long time to dissolve and probably responsible for the decrease of the reaction speed at the end of the reaction. The consequences of steric hindrance, i.e., the interaction between several grains and needles, points out the failure of a naive interpretation of calorimetry experiments consisting in assuming that the extent of the reaction would evolve as if a single monocrystal of gypsum was growing. In the literature, the typical S-shape associated with the time variation of the extent of the reaction is too often decomposed into an induction period related to plaster dissolution, a rapid increase associated with gypsum nucleation and a final relaxation related to gypsum growth $[16,47]$. In fact, the growth of many interacting gypsum needles and the dissolution of many plaster grains occur simultaneously throughout the reaction. The reaction involves the growth of a very large number of interacting crystals, for which statistical physics and stochastic processes offer well-adapted modeling tools. Conductivity measurements during plaster hydration also show a typical S-shape. However, the observed decrease of conductivity is related to the very end of the reaction when the remaining plaster is in too small a quantity to counterbalance the consumption of ions due to gypsum precipitation and to maintain the ion concentration constant. Consequently, the conductivity curves do not provide information on the main period during which gypsum precipitates and we we would like to warn against a misinterpretation of these experiments. 
In conclusion, this work provides precise criteria for optimizing the kinetics of plaster hydration and the structure of the material. The processes that affect the number of nucleation sites, the available surface area on which gypsum can precipitate and the impediments to needle growth due to steric hindrance offer good levers to control kinetics. We recommend to add gypsum germs with an $m_{g} / m_{p}=0.002$ gypsum-to-plaster mass ratio in order to optimize homogeneity of the final material and a priori improve the mechanical properties.

\section{Appendix: Simulation algorithm}

The simulation is based on a kinetic Monte Carlo algorithm [19, 22, 23, 43, 44]. The plaster grains are modeled by spheres of variable radius $R$, that are randomly spread in a cubic box of side $L$ with periodic boundary conditions. The experimental grain size distribution assigns a physical length scale to the simulation unit of length. The grains are placed in decreasing order of radii with a non-overlapping constraint. The plaster paste is characterized by a given water-to-plaster mass ratio, $m_{w} / m_{p}$, where $m_{w}$ and $m_{p}$ are the masses of water and plaster, respectively. We admit that nuclei preexist on the surface of the plaster grains when the reaction of hydration begins. At the beginning of the simulation, these nuclei are randomly placed on the grains with a surface density $g$. The nuclei are supposed to already have the shape of embryonic gypsum needles. They are rectangular parallelepipeds of length $l_{0}$, of the order of a few nanometers, and with a square base of side $l_{0} / \alpha_{0}$, where $\alpha_{0}$ is the initial aspect ratio. Moreover, some micrometric gypsum needles may be added to the water with different gypsum-to-plaster mass ratios $m_{g} / m_{p}$, where $m_{g}$ is the mass of gypsum seeds initially introduced. The gypsum seeds introduced in the bulk are parallelepipedic needles of length $l_{0}^{\prime}$ with the same initial aspect ratio $\alpha_{0}$ as the nucleation sites on the surface of the grains. The direction of the needles are randomly chosen. The total number of needles and their directions remain unchanged during the simulation. 
The kinetic Monte Carlo procedure requires the definition of the rates of gypsum needle growth and plaster grain shrinking. During the simulation time step $\Delta t$, the growth of each needle is considered. The time step is assumed to be long enough for a growing needle to recover a proper aspect ratio. We introduce a constant length increase $\Delta l_{0}$ and width increase $\Delta l_{0} / \alpha_{0}$ and assume that, initially, the growth probability of a needle is proportional to its surface $s$. Hence, two different needle growth regimes are considered: Growth is first accepted with a surface-dependent probability $p s / s_{c}$, until needle surface $s$ reaches the critical value $s_{c}$. Then, growth occurs with the constant probability $p$ imposed by a diffusion-limited rate [39]. Actual needle growth is accepted if the faces are not blocked by steric hindrance, i.e., the contact with other solids, such as plaster grains and other needles. We refer to reference [44] for a detailed presentation of the algorithm that we implemented to check the contacts between parallelepipeds and spheres. If growth is allowed, a same volume $w^{2} \Delta l_{0}$ of gypsum is deposited on each free face, where $\Delta l_{0}$ is constant. Purely homothetic growth, i.e., with strict conservation of the aspect ratio, occurs if none of the pairs of opposite faces is blocked. Even in the case of blocked opposite faces, the growth of a free face is accepted if the aspect ratio of the needle is in the allowed interval. On the simulation time scale, the growth process is irreversible and the needles never shrink.

After needle growth, grain shrinking is systematically performed according to the conservation of matter. More precisely, we compute the increase $\Delta V_{g}(t)=V_{g}(t+\Delta t)-$ $V_{g}(t)$ of the total volume of gypsum during time $t$ and $t+\Delta t$ as the sum of the volumes precipitated during $\Delta t$ on the needles for which growth has been accepted. The number of moles of gypsum that precipitated is equal to $n_{g}(t)=\Delta V_{g}(t) \frac{\rho_{g}}{M_{g}}$ where $\rho_{g}$ is the density of gypsum and $M_{g} \mathrm{~kg}$ is the molar mass of gypsum or dihydrate. Consequently, a same number of moles of plaster has to be dissolved and the total volume of plaster to be 
dissolved is:

$$
\Delta V_{p}(t)=\Delta V_{g}(t) \frac{\rho_{g}}{\rho_{p}} \frac{M_{p}}{M_{g}}
$$

where $\rho_{p}$ and $M_{p} \mathrm{~kg}$ are the density and molar mass of plaster or hemihydrate, respectively. We assume that all the plaster grains loose an external shell of the same thickness $\Delta R(t)$ :

$$
\Delta R(t)=\frac{\Delta V_{p}(t)}{4 \pi \sum_{k=1}^{N} R_{k}^{2}}
$$

where $R_{k}$ is the radius of the plaster grain $k$ and where the summation is performed over all the $N$ grains existing at time $t$. As a consequence, small grains will rapidly disappear from the mixture whereas big grains may remain until the end of the reaction, possibly resulting in an incomplete reaction [23].

After a succession of growth and dissolution steps, the simulation ends either when the total amount of plaster has dissolved or when all the needles are blocked by steric hindrance.

\section{References}

[1] C. Rinaudo, M. C. Robert, F. Lefaucheux, Growth and Characterization of Gypsum Crystals, J. Cryst. Growth 1985, 71, 803-806.

[2] L. Amathieu, R. Boistelle, Improvement of the Mechanical Properties of Set Plasters by Means of Four Organic Additives Inducing $\{101\}$ Faces, J. Cryst. Growth 1986, 79, 169-177.

[3] C. Rinaudo, M. Franchini-Angela, R. Boistelle, Gypsum Crystallization from Cadmium-Poisoned Solutions, J. Cryst. Growth 1988, 89, 257-266.

[4] C. H. De Vreugd, G. J. Witkamp, G. M. van Rosmalen, Growth of Gypsum III. Influence and Incorporation of Lanthanide and Chromium Ions, J. Cryst. Growth 1994, $144,70-78$. 
[5] C. Rinaudo, A. M. Lanfranco, R. Boistelle, The Gypsum-Brushite System - Crystallization from Solutions Poisoned by Phosphate Ions, J. Cryst. Growth 1996, 158, 316-321.

[6] D. Bosbach, J. L. Junta-Rosso, U. Becker, M. F. Hochella Jr, Gypsum Growth in the Presence of Background Electrolytes Studied by Scanning Force Microscopy, Geochimica et Cosmochimica Acta 1996, 60, 3295-3304.

[7] E. Badens, S. Veesler, R. Boistelle, Crystallization of Gypsum from Hemihydrate in Presence of Additives, J. Cryst. Growth 1999, 198/199, 704-709.

[8] J.-P. Boisvert, M. Domenech, A. Foissy, J. Persello, J.-C. Mutin, Hydration of Calcium Sulfate Hemihydrate $\left(\mathrm{CaSO}_{4}, 1 / 2 \mathrm{H}_{2} \mathrm{O}\right)$ into Gypsum $\left(\mathrm{CaSO}_{4}, 2 \mathrm{H}_{2} \mathrm{O}\right)$. The Influence of the Sodium Polyacrylate/Surface Interaction and Molecular Weight, J. Cryst. Growth 2000, 220, 579-591.

[9] F. Brandt, D. Bosbach, Bassanite $\left(\mathrm{CaSO}_{4}, 0.5 \mathrm{H}_{2} \mathrm{O}\right)$ Dissolution and Gypsum $\left(\mathrm{CaSO}_{4}\right.$, $\left.2 \mathrm{H}_{2} \mathrm{O}\right)$ Precipitation in the Presence of Cellulose Ethers. J. Cryst. Growth 2001, 233, $837-845$.

[10] Hill, J.-R.; Plank, J. Retardation of Setting of Plaster of Paris by Organic Acids: Understanding the Mechanism through Molecular Modeling. J. Comput. Chem. 2004, 25, 1438-1448.

[11] M. M. Rashad, M. H. H. Mahmoud, I. A. Ibrahim, E. A. Abdel-Aal, Crystallization of Calcium Sulfate Dihydrate under Simulated Conditions of Phosphoric Acid Production in the Presence of Aluminum and Magnesium Ions, J. Cryst. Growth 2004, 267, 372379.

[12] P. Reynaud, M. Saâdaoui, S. Meille, G. Fantozzi, Water Effect On Internal Friction Of Set Plaster, Mater. Sci. Eng. A 2006, 442, 500-503. 
[13] A. Ersen, A. Smith, T. Chotard, Effect of Malic and Citric Acid on the Crystallisation of Gypsum Investigated by Coupled Acoustic Emission and Electrical Conductivity Techniques, J. Mater. Sci. 2006, 41, 7210-7217.

[14] E. M. Gartner, Cohesion and Expansion in Polycrystalline Solids Formed by Hydration Reactions The Case of Gypsum Plasters, Cem. Concr. Res. 2009, 39, 289-295.

[15] P. Dalmay, A. Smith, T. Chotard, P. Sahay-Turner, V. Gloaguen, P. Krausz, Properties of Cellulosic Fibre Reinforced Plaster: Influence of Hemp or Flax Fibres on the Properties of Set Gypsum, J. Mater. Sci. 2010, 45, 793803.

[16] A. Gmouh, S. Eve, A. Samdi, R. Moussa, J. Hamel, M. Gomina, Changes in plaster microstructue by pre-stressing or by adding gypsum grains: microstructural and mechanical investigations, Mater. Sci. Eng. A 2003, 352, 325-332.

[17] W. Prakaypun, S. Jinawath, Comparative effect of additives on the mechanical properties of plasters made from flue-gas desulfurized, and natural gypsum. Mater. Struct. 2003, 36, 51-58.

[18] L. Amathieu, R. Boistelle, Crystallization Kinetics of Gypsum from Dense Suspension of Hemihydrate in Water, J. Cryst. Growth 1988, 88, 183-192.

[19] G. Dumazer, V. Narayan, A. Smith, A. Lemarchand, Modeling Gypsum Crystallization on a Submicrometric Scale, J. Phys. Chem. C 2009, 113, 1189-1195.

[20] A. J. Lewry, J. Williamson, The Setting of Gypsum Plaster, Part I, The Hydration of Calcium Sulphate Hemihydrate, J. Mater. Sci. 1994, 29, 5279-5284.

[21] C. Martias, Y. Joliff, J. Rogez, C. Favotto, A new composite based on gypsum matrix and mineral additives: Hydration process of the matrix and thermal properties at room temperature, Thermochim. Acta 2013, 567, 15-26. 
[22] A. Lemarchand, F. Boudoire, E. Boucard, T. Chotard, A. Smith, Plaster Hydration at Different Plaster-to-Water Ratios: Acoustic Emission and 3-Dimensional Submicrometric Simulations, J. Phys. Chem. C 2012, 116, 4671-4678.

[23] S. Joiret, F. Pillier, A. Lemarchand, Submicrometric Picture of Plaster Hydration: Dynamic and Space-Resolved Raman Spectroscopy versus Kinetic Monte Carlo Simulations, J. Phys. Chem. C 2014, 118, 28730-28738.

[24] Y.-W. Wang, Y.-Y. Kim, H. K. Christenson, F. C. Meldrum, A New Precipitation Pathway for Calcium Sulfate Dihydrate (Gypsum) via Amorphous and Hemihydrate Intermediates, Chem. Commun. 2012, 48, 504-506.

[25] A. E. S. Van Driessche, L. G. Benning, J. D. Rodriguez-Blanco, M. Ossorio, P. Bots, J. M. Garcia-Ruiz, The Role and Implications of Bassanite as a Stable Precursor Phase to Gypsum Precipitation, Science 2002, 336, 69-72.

[26] K. M. Song, J. Mitchell, H. Jaffel, L. F. Gladden, Simultaneous Monitoring of Hydration Kinetics, Microstructural Evolution, and Surface Interactions in Hydrating Gypsum Plaster in the Presence of Additives, J. Mater. Sci. 2010, 45, 5282-5290.

[27] A. Saha, J. Lee, S. M. Pancera, M. F. Bräeu, A. Kempter, A. Tripathi, A. Bose, New Insights into the Transformation of Calcium Sulfate Hemihydrate to Gypsum Using Time-Resolved Cryogenic Transmission Electron Microscopy, Langmuir 2012, 28, 11182-11187.

[28] E.J. Garboczi, K. A. Snyder, J. F. Douglas, M. F. Thorpe, Geometrical Percolation Threshold of Overlapping Ellipsoids, Phys. Rev. E 1995, 52, 819-828.

[29] S. Meille, E. J. Garboczi, Linear Elastic Properties of 2-D and 3-D Models of Porous Materials Made From Elongated Objects, Modelling Simul. Mater. Sci. Eng. 2001, 9, 371-390. 
[30] J. Sanahuja, L. Dormieux, S. Meille, C. Hellmich, A. Fritsch, Micromechanical Explanation of Elasticity and Strength of Gypsum: From Elongated Anisotropic Crystals to Isotropic Porous Polycrystals, J. Eng. Mech. 2010, 136, 239253.

[31] Q. L. Yu, H. J. H. Brouwers, Microstructure and Mechanical Properties of $\beta$ Hemihydrate Produced Gypsum: An Insight from its Hydration Process, Constr. Build. Mater. 2011, 25, 3149-3157.

[32] Q. Ye, B. Guan, Y. Lou, B. Kong, Effect of particle size distribution on the hydration and compressive strength development of $\alpha$-calcium sulfate hemihydrate paste, Powder Technol. 2011, 207, 208-214.

[33] D. Jeulin, P. Monnaie, F. Péronnet, Gypsum morphological analysis and modeling, Cement $\&$ Concrete Composites 2001, 23, 299-311.

[34] G. M. Rosmalen, P. J. Daudey, W. G. J. Marchée, An Analysis of Growth Experiments of Gypsum Crystals in Suspension, J. Cryst. Growth 1981, 52, 801-811.

[35] J. Beretka, J. W. van der Touw, Hydration Kinetics of Calcium Sulphate Hemihydrate: A Comparison of Models, J. Chem. Tech. Biotechnol. 1989, 44, 19-30.

[36] R. J. Hand, The Kinetics of Hydration of Calcium Sulphate Hemihydate: A Critical Comparison of the Models in the Literature, Cem. Concr. Res. 1994, 24, 885-895.

[37] A. Hernandez, A. La Rocca, H. Power, U. Graupner, G. Ziegenbalg, Modelling the Effect of Precipitation Inhibitors on the Crystallization Process of an Over-Saturation Solution in Gypsum in a Stirring Experiment, J. Cryst. Growth 2006, 295, 217-230.

[38] E. Finot, E. Lesniewska, J.-P. Goudonnet, J.-C. Mutin, M. Domenech, A. Aït Kadi, Correlating surface forces with surface reactivity of gypsum crystals by atomic force microscopy. Comparaison with rheological properties of plaster, Solid State Ionics 2001, 141-142, 39-46. 
[39] A. E. S. Van Driessche, J. M. Garcia-Ruiz, J. M. Delgado-Lopez, G. Sazaki In Situ Observation of Step Dynamics on Gypsum Crystals, Cryst. Growth Des. 2010, 10, 3909-3916.

[40] F. Jones, Infrared investigation of barite and gypsum crystallization: Evidence for an amorphous to crystalline transition CrystEngComm 2012, 14, 8374-8381.

[41] R. K. Mishra, PhD thesis of the University of Akron (USA), Simulation of interfaces in construction materials : Tricalcium silicate, gypsum, and organic modifiers (2012).

[42] T. M. Stawski, A. E. S. van Driessche, M. Ossorio, J. D. Rodriguez-Blanco, R. Besselink, L. G. Benning, Formation of calcium sulfate through the aggregation of sub-3 nanometre primary species, Nature Comm. 2016, 7, 11177.

[43] G. Dumazer, A. Smith, A. Lemarchand, Master Equation Approach to Gypsum Needle Crystallization, J. Phys. Chem. C 2010, 114, 3830-3836.

[44] G. Morgado, M. Collet, R. Lespiat, H. Rétot, A. Lemarchand, Submicrometric Picture of Plaster Hydration: Optimization of the Addition of Gypsum Needles, J. Phys. Chem. C 2017, 121, 5657-5666.

[45] G. Nicolis and I. Prigogine, Self-Organization in Nonequilibrium Systems, Wiley, New York (1977).

[46] C. W. Gardiner, Handbook of Stochastic Methods, Springer, Berlin (1985).

[47] N. B. Singh, B. Middendorf, Calcium sulphate hemihydrate hydration leading to gypsum crystallization, Prog. Cryst. Growth Charact. Mater. 2007, 53, 57-77.

[48] J. A. Venables, Introduction to Surface and Thin Film Processes, Cambridge University Press, Cambridge, UK (2000).

[49] B. Fritz, A. Clément, Y. Amal, C. Noguera, Simulation of the nucleation and growth of simple clay minerals in weathering processes: the NANOKIN Code, Geochim. Cosmochim. Acta 2009, 73, 1340-1358. 
[50] B. Fritz, C. Noguera, Mineral Precipitation Kinetics, Rev. Mineral. Geochem. 2009, 70, 371-410.

[51] J.-F. Georgin, N. Maach, J. Pommay, Sulphate crystallization modelling and surface reactivity in solution, Constr. Build. Mater. 2017, 156, 266-276.

[52] J.-P. Salvetat-Delmotte, A. Rubio, Mechanical properties of carbon nanotubes: a fiber digest for beginners, Carbon 2002, 40, 1729-1734. 